\title{
«Prevention is better than healing»
}

\section{Dominik Heim}

PD Dr. med., Kompetenzzentrum für Versicherungsmedizin, Suva, Luzern

\author{
Die SITEMSH, Société Internationale de Traumatologie du Ski et de Médecine des
} Sports d'Hiver, wurde in den 50er Jahren aus dem Bedürfnis heraus gegründet, sich dem zunehmenden Problem von Wintersportverletzungen zu stellen. Sie hatte grossen Zulauf in den Alpenländern. Man gehörte einfach dazu! Trotz zunehmender Spezialisierung in der Traumatologie blieb sie bestehen, weil sie sich dem Wintersportunfall als Ganzem mit all seinen Aspekten von Rettung, Transport, Therapie und Prävention widmet. Jetzt kommt der SITEMSH-Kongress nach vielen Jahren wieder in die Schweiz.

Ein wichtiger Vertreter aus der Schweiz in der SITEMSH war Prof. Hans-Ulrich Buff (1913-2004), der Chefarzt der Chirurgischen Klinik für Traumatologie und Wiederherstellungschirurgie am Zürcher Universitätsspital. Er erwarb sich mit seiner breiten chirurgischen und akademischen Tätigkeit international grosse Anerkennung und erhielt zahlreiche nationale und internationale Ehrungen (u.a. Mitglied der Académie de Chirurgie de Paris). Er war auch ein begeisterter Skiund Ferrari-Fahrer. Er wurde nach Robert Merle d'Aubingé (1900-1989), dem weltberühmten französischen Orthopäden, der zweite Präsident der SITEMSH. «Buff war der Meinung, kein Englisch, sondern man vertraue auf das gegenseitige Sprachverständnis der Alpenländer. In Wirklichkeit setzte er seine Fähigkeit ein, die Referate prägnant zusammenzufassen und

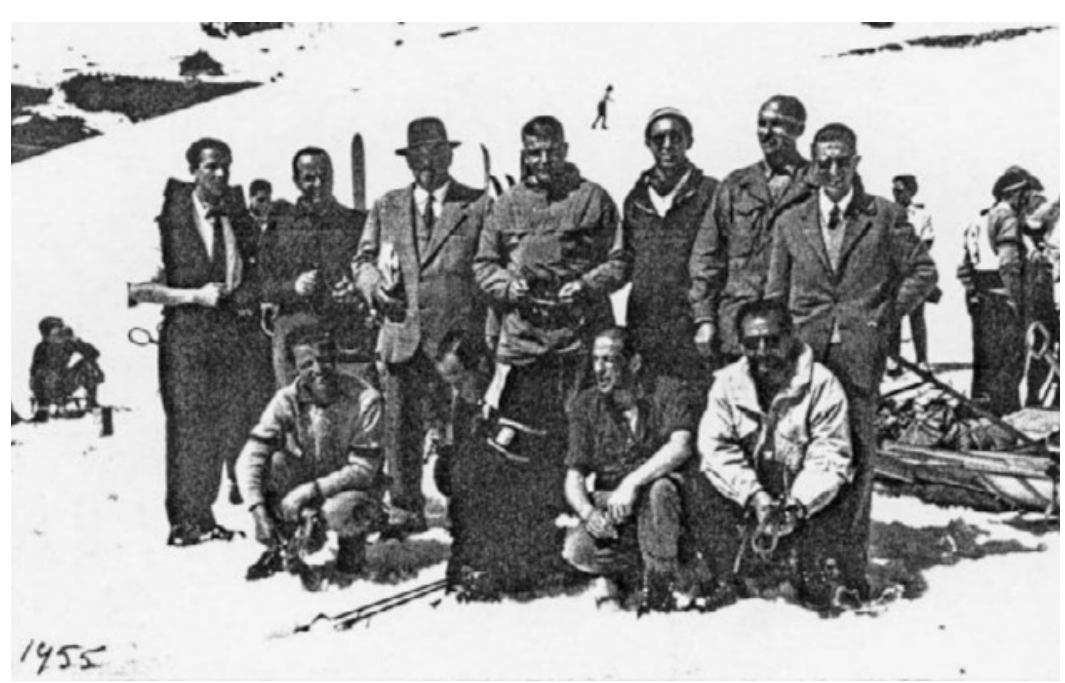

Abbildung 1: Die nationalen Sekretäre der 1956 gegründeten SITEMSH an einem Treffen 1955. in die jeweils andere Sprache zu übersetzen», erzählt Klaus Herwig. Herwig seinerseits war ab 1971 der internationale Sekretär der SITEMSH und Nachfolger von Franz Jakob, Chefarzt des Spitals Davos.

\section{Die Geschichte}

Gegründet wurde die Gesellschaft am 29. Dezember 1956: «Sous le nom de SITEMSH, Société Internationale de Traumatologie du Ski et de Médecine des Sports d'hiver, une association a été constituée selon l'article 60 et suivants du Code Civil Suisse à la date du 29 Décembre 1956", steht in der Statuten-Version vom 13. April 1991, die in Andorra von den damaligen nationalen Sekretären unterzeichnet wurde. Wobei Statuten eigentlich als unwichtig angesehen worden seien, wie Klaus Herwig präzisiert. Es sei darum gegangen, alle zwei Jahre einen wissenschaftlichen Kongress zum Wintersport durchzuführen.

Und diese Kongresse fanden denn auch regelmässig statt: 1954 in Courchevel, 1956 in Sestrière, 1958 in Davos, und so ging es alle zwei Jahre weiter. 112 Teilnehmer waren es 1978 beim 13. Kongress in Arosa. Die SITEMSH gilt damit als die älteste europäische medizinische Gesellschaft, die sich mit den Wintersportverletzungen, ihren Behandlungen und ihrer Prävention befasst. Im historischen Rückblick der SITEMSH 2014 liest man dazu «In the '50s a group of experts of the Alpine Countries (Austria, France, Germany, Italy and Switzerland) began to observe, collect and study the injuries coming from ski practice. They decided to meet themselves every two years» (Abb. 1).

Unumstritten war diese Gesellschaft nicht. Fritz Lechner sagte in seiner Eröffnungsansprache am 9. Inter- 
nationalen Kongress in Garmisch-Partenkirchen 1970: «Bei der Flut der heutigen Kongresse und Tagungen könnte mit Recht die Frage nach dem Sinn und der Berechtigung dieser Veranstaltung gestellt werden. Themen der Unfallchirurgie wurden in den letzten Jahren regelmässig auf den Kongressen der internationalen und nationalen Gesellschaften für Chirurgie und Unfallchirurgie behandelt. Die Probleme der Skitraumatologie und Wintersportmedizin sind aber von besonderer Art. Sie beginnen bei der Bergung und beim Abtransport der Skiverletzten und enden mit der Diagnostik und Behandlung in der Klinik ... Zudem ist die Gesellschaft eine internationale Familie von Chirurgen, die sich zum grossen Teil bereits persönlich kennen und freundschaftlich miteinander verbunden sind.» Dies betont auch Franz Jakob in seinem Übergabebrief an Klaus Herwig: "Wie Sie wissen, herrscht immer ein sehr offener, freundschaftlicher Ton.» Und er fügt bei: "Als internationaler Sekretär werden von Ihnen keine grossen oratorischen Künste erwartet!»

\section{Das Skirennen}

Und da gab es noch etwas Spezielles: das traditionelle Skirennen der Kongressteilnehmer, die sogenannte «Coupe du président» - $1974 \mathrm{im}$ Val d'Isère waren es 130 Teilnehmer aus ganz Europa. Im 2. Rang war ein gewisser Maurice E. Müller (AO-Gründungsmitglied und ehemaliger Ordinarius am Inselspital), gefolgt von Hans Ulrich Buff vor Martin Allgöwer (AO-Gründungsmitglied und ehemaliger Ordinarius am Universitätsspital Basel sowie «Erfinder» der Donati-AllgöwerHautnaht-Technik)! Selbstverständlich nahmen an den Rennen auch die Damen der Kongressteilnehmer teil. Herwig sagt dazu: «Des öfteren wurden gar keine Kongressberichte erstellt. Statt Teilnehmerlisten existieren Ranglisten von Skirennen.» Und noch etwas war speziell: Geld hatte die Gesellschaft nie: 1967 berichtet Franz Jakob an der Vorstandssitzung von 1380.93 Franken, "so dass eine Beihilfe an die Finanzierung des Kongresses in St. Moritz nicht möglich sein wird». Das ist heute nicht anders!

\section{Die Gegenwart}

1974 wurde dann eine weitere Gesellschaft, die ISSS (International society for skiing safety), gegründet. An den Kongressen der jeweiligen Gesellschaft nehmen häufig Mitglieder beider Gesellschaften teil.

Und die SITEMSH wurde immer internationaler, aber auch immer kleiner. Neben den erwähnten Gründerländern stiessen Andorra (1990), Spanien (1993), Chile (2007), Griechenland (2008), Argentinien, die USA, Schottland, Tschechien und Ungarn dazu. Ganz neu sind nationale Sekretäre aus Japan, Iran, Kasachstan und China. Heute zählt die SITEMSH 18 Mitgliederlän-

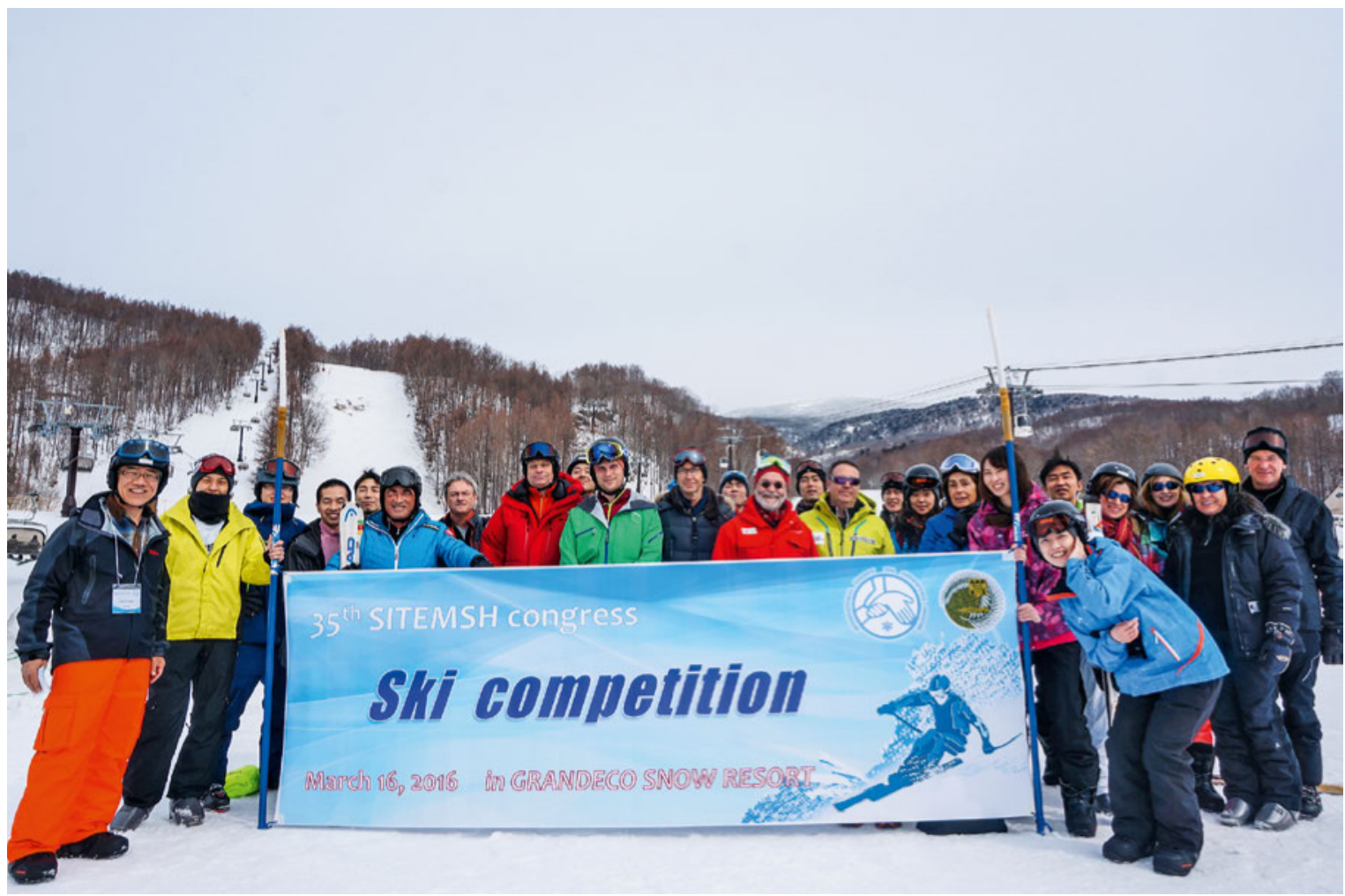

Abbildung 2: Die «Coupe du président» 2016 in Inawashiro, Japan. 


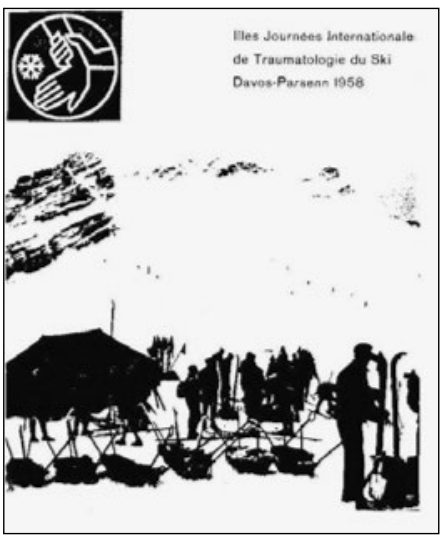

Abbildung 3: Das Ankündigungsplakat des Kongresses 1958 in Davos.

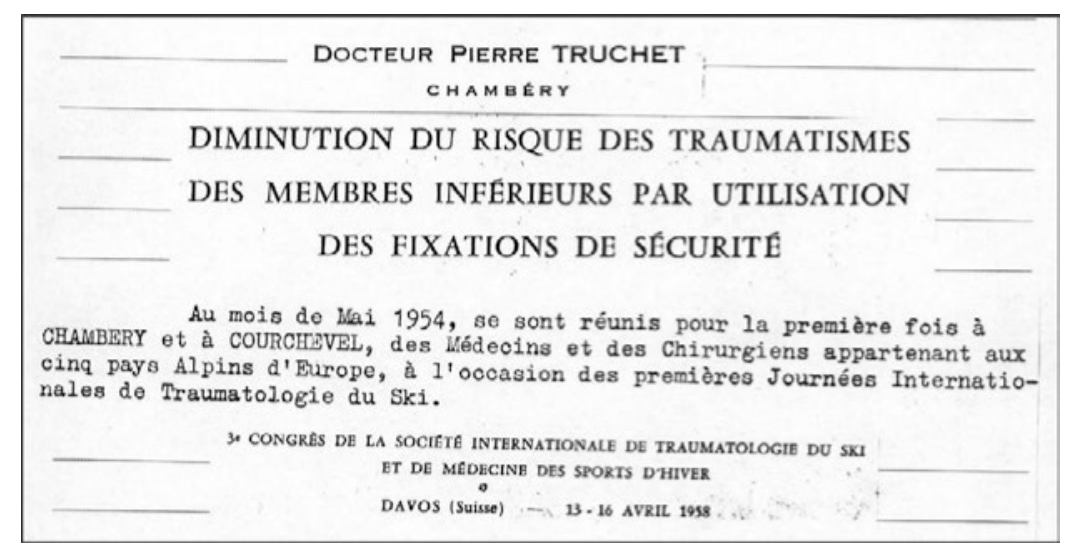

Abbildung 4: Das Thema der Sicherheitsbindung am Kongress 1958 in Davos. der und nennt sich International Society for Snowsports Medicine. Und die Skirennen finden immer noch statt (Abb. 2).

35 Kongresse wurden bis heute in verschiedenen Ländern durchgeführt. In der Schweiz war dies zum letzten Mal 1998 in Zermatt der Fall.

Durch diese lange Zeit ihrer Aktivität verfügt die SITEMSH über einen umfassenden Überblick über die Wintersporttraumatologie. Ihre Kongresse und Kongressthemen in den letzten rund 60 Jahren reflektieren die Entwicklung im Schneesport, im Verletzungsmuster und in der Prävention. In Davos 1958 (Abb. 3) war das grosse Thema die neue Sicherheitsbindung (Abb. 4).

Die weitere, neuere Geschichte kennen wir: Die Unterschenkelfrakturen nahmen dank der Sicherheitsbindung rasant $a b$, und die Knieverletzungen nahmen rasant zu. Es folgte die Verlagerung der Verletzungen von der unteren Extremität [1] zur oberen Extremität. Die Snowboardverletzungen kamen hinzu und trugen zu dieser Verschiebung bei. In den letzten Jahren erlebte der Schlittelsport ein Comeback und brachte ein neues Verletzungsmuster mit sich, das eher wieder die untere Extremität «bevorzugt». Vor allem zeigen diese Verletzungen einen ziemlichen Schweregrad [2]. Das Neurotrauma wurde ein grosses Thema. Die Kniefrakturen werden immer komplizierter, und angesichts der gut präparierten Pisten spricht man von einem eigentlichen Geschwindigkeitsrausch. Kollisionen machen im heutigen Wintersport sieben bis zehn Prozent der Unfallursachen aus. Verschiedene nationale Präventionskampagnen finden/fanden statt: Der Helm ist überall präsent, man trägt beim Freestylen einen Rückenpanzer, man schützt sein Handgelenk mit Manschetten und Schonern, und spezielle Vorrichtungen sollen bei Lawinenunglücken helfen. Aber bei all diesen Bemühungen ist das Problem des Knies mit seinen desaströsen Binnenläsionen weiterhin ungelöst. Vielleicht gelingt es am 36. SITEMSH-Kongress in Arosa, neue biomechanische Ansätze zu erarbeiten. Ganz neue Erkenntnisse aus den USA lassen dies erhoffen, und damit würde dieser Kongress 2018 fast nahtlos an jenen in Davos vor 60 Jahren anschliessen.

Dank

Ich danke Herrn Dr. med. Klaus Herwig, Arosa, für seine wichtigen Informationen und für das Überlassen der frühen Dokumente.

\section{Bildnachweis}

Abdruck des Gruppenbilds 2016 (Inawashiro, Japan) mit freundlicher Genehmigung von Dominik Heim / Yukio Urabe.

Abdruck der übrigen Fotos/Abbildungen mit freundlicher Genehmigung der SITEMSH.

\section{Literatur}

1 Heim D. Tendenzen im Verletzungsmuster des alpinen Wintersports. Praxis. 1997;86:1931-3.

2 Heim D, Altgeld K, Hasler RM, et al. A 13-year analysis from Switzerland of non-fatal sledging (sledding or tobogganing) injuries. Injury, Int. J. Care Injured. 2014;45:338-41.

\section{Der SITEMSH-Kongress 2018 in Arosa}

Der 36. SITEMSH-Kongress findet vom 13.-15. März 2018 im Kongresszentrum Arosa statt. Weitere Hinweise und das Kongressprogramm sind auf der Website der SITEMSH www.sitemsh.org einzusehen.

Kongressregistrierung in der Schweiz: edithrubin[at]bluewin.ch 